\title{
Long-Term Quality of Life of Melanoma Survivors Is Comparable to that of the General Population
}

\author{
PIA J. HEINO ${ }^{1}$, PIA H. MYLLÄRI ${ }^{1}$, TIINA A. JAHKOLA ${ }^{1}$, HARRI SINTONEN ${ }^{2}$, \\ MINNA-LIISA LUOMA ${ }^{3}$, PIRJO RÄSÄNEN ${ }^{4}$ and RISTO P. ROINE ${ }^{5}$ \\ ${ }^{1}$ Department of Plastic and Reconstructive Surgery, \\ University of Helsinki and Helsinki University Hospital, Helsinki, Finland; \\ ${ }^{2}$ Department of Public Health, University of Helsinki, Helsinki, Finland; \\ ${ }^{3}$ Ageing, Disability and Functioning Unit, National Institute for Health and Welfare, Helsinki, Finland; \\ ${ }^{4}$ Hospital District of Helsinki and Uusimaa, Helsinki, Finland; \\ ${ }^{5}$ University of Helsinki and Helsinki University Hospital, and University of Eastern Finland, Kuopio, Finland
}

\begin{abstract}
Background/Aim: There is a growing need for information regarding the Health-Related Quality of Life (HRQoL) of cancer survivors. This study aimed to assess the HRQoL of patients treated for cutaneous malignant melanoma between 1980 and 2004 in the Helsinki and Uusimaa Hospital district and compare the results to the general population. Materials and Methods: HRQoL of 981 cutaneous melanoma patients (aged 13 to 97 years, 56.1\% female) was assessed using the generic $15 D$ instrument and compared to the general population. The association between demographic and clinical factors and HRQoL was analyzed using oneway ANOVA, student's t-test and multivariate regression. Results: The mean $15 D$ score of melanoma patients was slightly lower (0.904) than that of the general population $(0.911, p=0.027)$, but the difference was not statistically significant. HRQoL deteriorates with age and metastatic disease and improves with time. Conclusion: No evidence was found that long-term HRQoL of melanoma survivors was worse than the general population.
\end{abstract}

The incidence of cutaneous malignant melanoma continues to rise dramatically in Western countries. In Finland, cutaneous melanoma is the most rapidly increasing cancer with a mean yearly incidence increase of 3.8\% among men and $6.3 \%$ among women during the past decade. Despite the

Correspondence to: Pia J. Heino, Department of Plastic and Reconstructive Surgery, P.O. Box 22, 00014 University of Helsinki and Helsinki University Hospital, Finland. Tel: +358503506718 , email: pia.heino@helsinki.fi

Key Words: Health-related quality of life, health status, health status indicators, melanoma, cutaneous, skin cancer, quality of life. increase in incidence, melanoma mortality has remained stable due to early diagnosis and surgical treatment $(1,2)$.

Melanoma treatment varies according to disease stage. Stage 1-2 melanoma (localized disease) is treated by excising the melanoma. The excision margin is chosen according to the thickness of the lesion. In the 1980's, the primary tumor was excised with a large tissue margin, which led to the need of skin transplantations or tissue flaps. It was later discovered that larger tissue margins do not lead to improved survival rates $(3,4)$. During the 1990's the recommended excision margins diminished significantly, thus reducing the potentially disfiguring effects of surgery. Another change in surgical treatment has been the development of sentinel lymph node biopsy (SNB), which was first introduced to the Finnish national treatment guidelines (5) in 2005.

Even with the introduction of sentinel - node biopsy and complete lymph node dissection, as well as new systemic drugs into melanoma treatment guidelines, the primary treatment of melanoma remains surgery. Approximately $84 \%$ of melanoma cases are at a localized stage at the time of diagnosis, for which the 5-year survival rate is $98 \%$ (6). The improved survival of melanoma patients has created a need for real-world quality of life data to better understand how patients cope with the disease and its treatments in the long term. A systematic review published in 2016 identified several studies attempting at identifying some of the factors that are associated with different levels of HRQoL of melanoma patients, however, the results across studies remained inconsistent (7). Consequently, more information is needed concerning the health-related quality of life (HRQoL) of melanoma patients and the association between different patient and disease characteristics and HRQoL.

Our study assessed, using the generic 15D HRQoL instrument, the HRQoL of melanoma patients diagnosed with 
melanoma between 1980 and 2004 in the Helsinki and Uusimaa Hospital district and compared the results to the HRQoL of the age- and gender-standardized general population. It also analyzed the association of some key demographic and clinical factors with HRQoL.

\section{Materials and Methods}

The study population and survey process. Our study population consists of patients diagnosed with melanoma between 1980 and 2004 in the catchment area of the Helsinki and Uusimaa Hospital District in Southern Finland. All patients diagnosed and treated for cutaneous melanoma between 1980 and 2004 in the area were eligible. The survey process is presented in Figure 1. Initially, we performed a keyword search. All hospital records, including outpatient clinic records, were scanned for patients with a cutaneous melanoma diagnosis. The keyword search yielded 2075 individuals with any mentioning of melanoma in their hospital records, out of whom 540 had died. Out of the remaining patients, 1381 individuals who were alive at the time of the survey with a confirmed melanoma diagnosis and a known address were identified. Questionnaires were sent to these patients during spring 2005. One reminder was sent in case of non-response. Out of the 1381 patients, 1052 returned the questionnaire (response rate $76.2 \%$ ), but nine of them had left it unanswered, out of which three because of Alzheimer's disease. Furthermore, 12 patients were excluded because they had left four or more questions of the 15D questionnaire unanswered and 59 patients because their clinical data could not be found from the patient registry. Thus, 981 patients were included in the final analysis.

Ethical aspects. The study was approved by the Ethics committee of the Helsinki University Central Hospital. Patients gave their written informed consent before inclusion.

The questionnaire. To study HRQoL we used the generic, 15dimensional, standardized, self-administered 15D instrument that can be used as both a profile and a single index score measure. The questionnaire includes the following dimensions: mobility, vision, hearing, breathing, sleeping, eating, speech, excretion, usual activities, mental functioning, discomfort and symptoms, depression, distress, vitality and sexual activity. For each dimension, the respondent chooses one of the five ordinal levels best describing his/her state of health at the moment (best value $=1$; worst value $=5$ ). The single index score (15D score), representing the overall HRQoL on a $0-1$ scale $(1=$ full health, $0=$ being dead $)$ and the dimension level values, reflecting the goodness of the levels relative to no problems on the dimension $(=1)$ and to being dead $(=0)$, are calculated from the questionnaire by using a set of population-based preference or utility weights. Mean dimension level values are used to draw 15D profiles for groups (8). The minimum clinically important change or difference in the $15 \mathrm{D}$ score has been estimated to be \pm 0.015 on the basis that people can on average feel such a difference (9). As the $15 \mathrm{D}$ is a generic instrument, i.e., not created specifically for cancer patients, it can be easily used to compare the HRQoL of patients to that of the general population. In addition to the $15 \mathrm{D}$ questionnaire, the patients were also asked to fill in the EORTCQLQ-C30 questionnaire. The results of the latter are however not discussed in this paper.

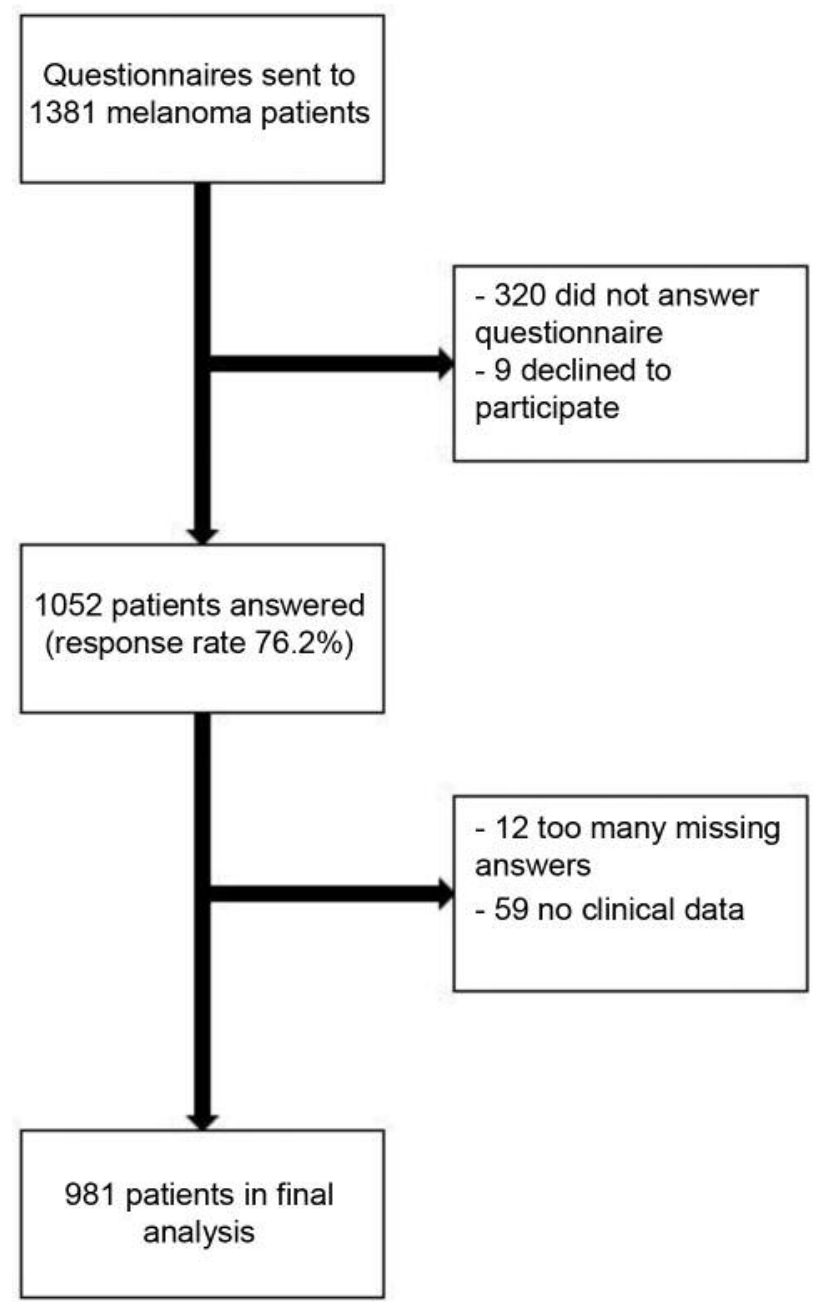

Figure 1. Patient flow chart.

Statistical analysis. The statistical analyses were performed using IBM SPSS Statistics version 24, STATA and LIMDEP version 7.0 (Greene WH. LIMDEP Version 7.0: User's manual, revised version. Econometric Software, Inc.: New York, 1998). We started by performing descriptive statistical analysis for key demographic factors, clinical characteristics, and treatments and then continued by comparing the mean $15 \mathrm{D}$ scores for each demographic factor or clinical characteristic by independent samples $t$-test for comparison between two groups and one-way ANOVA for comparison between three or more groups.

The $15 \mathrm{D}$ results of the melanoma patients were compared, using the independent samples $t$-test, with those of a representative sample of the age- and gender-standardized general population in the Helsinki and Uusimaa Hospital District. This sample includes the participants in the stratum of that District in the Finnish Health 2011 Health Examination Survey (H2011 Survey). The participants in the H2011 Survey were the living participants in the Finnish Health 2000 Health Examination Survey (H2000 Survey), aged 29 and over, supplemented with a new sample aged 


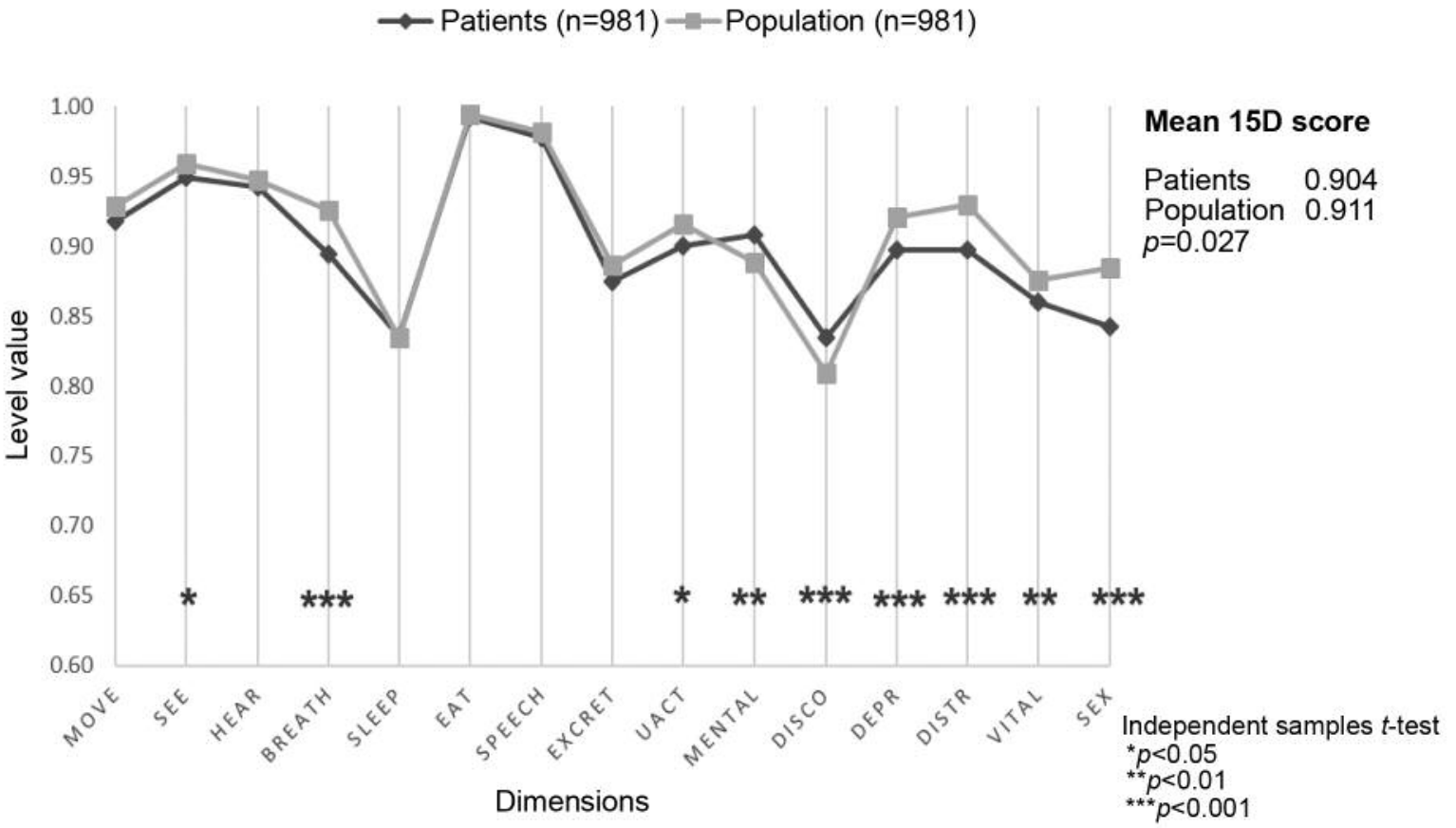

Figure 2. The mean 15D profile of melanoma patients compared to that of the age-and gender-standardized general population.

18-28. For this study, those participants from the Helsinki and Uusimaa District who were in the age range of the patients, were selected. This subsample was weighted to reflect the age and gender distribution of patients.

A multivariate statistical analysis was also performed. The variance in the $15 \mathrm{D}$ scores was explained by a Tobit regression model. The model was deemed suitable for two reasons. Firstly, the distribution of the residuals of the dependent variable (15D score) was not normal (according to the Kolmogorov-Smirnov test), but skewed and censored at 0 and 1 (the range of the scores is $0-1$ ) and, secondly, a substantial proportion of the observations was at the upper limit of 1 (18.3\%). Log-transformation of the data was also attempted, but the distribution of the residuals remained nonnormal. The Tobit model accounts for these special features of the distribution $(10,11)$.

The explanatory variables were chosen for the model on the basis of clinical relevance. The variance inflation factor was determined to detect possible severe multicollinearity. The variables chosen for the model were age, gender, time from surgery in years, known metastatic disease at the time of response, location of melanoma, excision margin, primary lymph node procedure (sentinel node biopsy or primary lymph node evacuation) and reconstruction technique (direct closure of wound, closure by skin graft or flap) The variables excision margin, melanoma location, lymph node procedure and surgical closure technique were transformed into categorical variables, where the first or lowest category is used as the comparison point. For excision margin, the cut-off points for each category were at $1.0 \mathrm{~cm}, 2.0 \mathrm{~cm}$, and $3.0 \mathrm{~cm}$. The cut-off points of $1.0 \mathrm{~cm}$ and $2.0 \mathrm{~cm}$ were chosen in accordance to current treatment guidelines, according to which the excision is performed with either margin depending on the thickness of the lesion. As it has been previously shown that a wider excision margin of $3.0 \mathrm{~cm}$ is associated with worse HRQoL (12), and a substantial proportion of the patients had been operated with a wider excision margin, the third cut-off point was set at $3.0 \mathrm{~cm}$. p-Values $<0.05$ were considered statistically significant.

\section{Results}

Characteristics of the study population. The characteristics of the study population are presented in Table I. Their mean age at the time of response was 60.5 years (range $=13-97$ years, SD 14.9) and did not differ markedly from that of the non-respondents, 58.3 years (range $=19-99$ years, SD 16.2 years). The proportion of female patients was $56.1 \%(n=550)$ (among non-respondents $53.5 \%$ ) and $2.8 \%$ of the study cohort had been diagnosed with metastatic disease at the time of answering the questionnaire. Information on time of primary surgery was obtained for 973 patients. The average time since primary surgery varied from 0 to 22 years with a mean of 6.1 (SD 4.7) and a median of 5.0 years. The mean $15 \mathrm{D}$ score for the whole study cohort was 0.904 (SD 0.103). The number of patients with valid data on their hospital records on each of the clinical variables gathered is presented in the column labeled "Valid" in Table I. The number of missing observations for each variable is presented in the adjacent column labeled "Missing". The results of the comparisons of 15D score means between groups are presented in Table I. 
Table I. Study population characteristics.

\begin{tabular}{|c|c|c|c|c|c|c|}
\hline Characteristic & $\mathrm{N}(\%)$ & SD & Valid & Missing $(\%)$ & Mean 15D (SD) & $p$-Value $\left(t\right.$-test/ANOVA) ${ }^{*}$ \\
\hline Age, mean (years, at the time of response) & 60.5 & 14.9 & 981 & $0(0)$ & $0.904(0.103)$ & \\
\hline Gender & & & 981 & $0(0)$ & & 0.128 \\
\hline Male $(\%)$ & $431(43.9)$ & & & & $0.905(0.109)$ & \\
\hline Female $(\%)$ & $550(56.1)$ & & & & $0.903(0.099)$ & \\
\hline Metastatic disease at the time of questionnaire & & & 979 & $2(0.2)$ & & 0.011 \\
\hline Yes & $27(2.8)$ & & & & $0.837(0.139)$ & \\
\hline No & $952(97.0)$ & & & & $0.906(0.101)$ & \\
\hline Time from primary surgical treatment (years) & 6.1 & 4.7 & 973 & $8(0.8)$ & & \\
\hline Ulceration & & & 896 & $85(8.7)$ & & 0.018 \\
\hline No & $811(82.7)$ & & & & $0.910(0.098)$ & \\
\hline Yes & $85(8.7)$ & & & & $0.864(0.124)$ & \\
\hline Breslow & & & 878 & $103(10.5)$ & & 0.006 \\
\hline$<1 \mathrm{~mm}$ & $381(38.8)$ & & & & $0.917(0.095)$ & \\
\hline $1-4 \mathrm{~mm}$ & $443(45.2)$ & & & & $0.896(0.105)$ & \\
\hline$>4 \mathrm{~mm}$ & $54(5.5)$ & & & & $0.893(0.120)$ & \\
\hline Clark & & & 948 & $33(3.4)$ & & $<0.001$ \\
\hline I & $87(8.9)$ & & & & $0.907(0.102)$ & \\
\hline II & $166(16.9)$ & & & & $0.919(0.092)$ & \\
\hline III & $348(35.5)$ & & & & $0.920(0.094)$ & \\
\hline IV & $326(33.2)$ & & & & $0.885(0.110)$ & \\
\hline $\mathrm{V}$ & $21(2.1)$ & & & & $0.883(0.106)$ & \\
\hline Excision margin & & & 961 & $20(2.0)$ & & 0.088 \\
\hline$<1 \mathrm{~cm}$ & $44(4.5)$ & & & & $0.872(0.123)$ & \\
\hline $1.00-1.99 \mathrm{~cm}$ & $396(40.4)$ & & & & $0.911(0.101)$ & \\
\hline $2.00-2.99 \mathrm{~cm}$ & $391(39.9)$ & & & & $0.900(0.102)$ & \\
\hline$\geq 3.00 \mathrm{~cm}$ & $130(13.3)$ & & & & $0.906(0.107)$ & \\
\hline Wound closure technique & & & 964 & $17(1.7)$ & & 0.461 \\
\hline Direct closure & $673(68.6)$ & & & & $0.906(0.102)$ & \\
\hline Skin graft & $151(15.4)$ & & & & $0.902(0.098)$ & \\
\hline Flap & $140(14.3)$ & & & & $0.895(0.113)$ & \\
\hline Primary location & & & 974 & $7(0.7)$ & & $<0.001$ \\
\hline Body & $400(40.8)$ & & & & $0.903(0.105)$ & \\
\hline Head and neck & $138(14.1)$ & & & & $0.883(0.111)$ & \\
\hline Upper limb & $171(17.4)$ & & & & $0.913(0.101)$ & \\
\hline Lower limb & $265(27.0)$ & & & & $0.910(0.098)$ & \\
\hline Primary lymph-node-procedure & & & 964 & $17(1.7)$ & & 0.123 \\
\hline No primary procedure & $653(66.6 \%)$ & & & & $0.909(0.101)$ & \\
\hline SNB & $231(23.5)$ & & & & $0.893(0.106)$ & \\
\hline Evacuation & $80(8.2)$ & & & & $0.898(0.105)$ & \\
\hline Complete lymph node dissection at any point & & & 963 & $18(1.8)$ & & 0.231 \\
\hline No & $782(79.7)$ & & & & $0.907(0.102)$ & \\
\hline Yes & $181(18.5)$ & & & & $0.894(0.105)$ & \\
\hline Stage & & & 387 & $594(60.5)$ & & 0.104 \\
\hline 0 & $39(4)$ & & & & $0.906(0.086)$ & \\
\hline I & $231(23.5)$ & & & & $0.913(0.094)$ & \\
\hline II & $53(5.4)$ & & & & $0.887(0.130)$ & \\
\hline III & $33(3.4)$ & & & & $0.908(0.081)$ & \\
\hline IV & $31(3.2)$ & & & & $0.879(0.119)$ & \\
\hline Primary complication & $58(6.0)$ & & 962 & $19(1.9)$ & $0.882(0.125)$ & 0.004 \\
\hline Wound infection & $26(2.7)$ & & & & & \\
\hline Seroma & $1(0.1)$ & & & & & \\
\hline Hematoma & $10(1.0)$ & & & & & \\
\hline Necrosis (wound or flap) & $9(0.9)$ & & & & & \\
\hline Failure in skin graft attachment & $2(0.2)$ & & & & & \\
\hline Wound opened & $9(0.9)$ & & & & & \\
\hline Facial nerve paresis & $1(0.1)$ & & & & & \\
\hline Lymph fistula & $1(0.1)$ & & & & & \\
\hline No primary complication & $904(92.2)$ & & & & $0.905(0.101)$ & \\
\hline
\end{tabular}


Table I. Continued

\begin{tabular}{|c|c|c|c|c|c|c|}
\hline Characteristic & $\mathrm{N}(\%)$ & SD & Valid & Missing (\%) & Mean 15D (SD) & $p$-Value ( $t$-test/ANOVA) \\
\hline Late complication & $14(1.4)$ & & 964 & $17(1.7)$ & $0.885(0.095)$ & 0.983 \\
\hline Seroma & $5(0.5)$ & & & & & \\
\hline Lymphedema & $6(0.6)$ & & & & & \\
\hline Recurrent erysipelas & $3(0.3)$ & & & & & \\
\hline Other & $1(0.1)$ & & & & & \\
\hline No late complication & $950(96.8)$ & & & & $0.904(0.103)$ & \\
\hline Local recurrence & & & 964 & $17(1.7)$ & & 0.095 \\
\hline No & 933 & & & & $0.905(0.102)$ & \\
\hline Yes & $31(3.2)$ & & & & $0.875(0.133)$ & \\
\hline Multiple melanomas & & & 965 & $16(1.6)$ & & 0.425 \\
\hline No & $929(94.7)$ & & & & $0.905(0.102)$ & \\
\hline Yes & $36(3.7)$ & & & & $0.892(0.120)$ & \\
\hline
\end{tabular}

*Comparison of means between groups using independent samples $t$-test/ one-way ANOVA.

Comparison with the general population. The melanoma patients had on average slightly lower overall HRQoL than the age-and gender-standardized general population $(\mathrm{p}=0.027)$, but the difference is not clinically important. On some dimensions (mental function and discomfort and symptoms) the patients seemed to be on average better off than the general population (Figure 2).

Multivariate analysis. The results of the regression model are presented in Table II. A total of 953 patients from the melanoma cohort were included in the multivariate analysis. The model showed a statistically significant coefficient for age, time from surgery and metastatic disease. An increase of one year in age lowers the $15 \mathrm{D}$ score by 0.003 points and a one-year increase in time from surgery increases the $15 \mathrm{D}$ score by 0.002 points. These numbers suggest that a fiveyear increase in age and an eight-year increase in the time from surgery, respectively, lower/increase the $15 \mathrm{D}$ score in a clinically important manner. The deteriorating effect of metastatic disease on HRQoL seems to be also clinically important. The variables of complication at any point after surgery and excision margin $>1 \mathrm{~cm}$ also had a clinically important, but statistically non-significant deteriorating effect on HRQoL. In this model, gender, reconstruction technique, anatomic location, primary sentinel node biopsy or complete lymph node dissection did not have a statistically significant effect on the $15 \mathrm{D}$ scores.

\section{Discussion}

In this cross-sectional observational study, we examined the association of the HRQoL of melanoma patients with some key clinical and demographic factors. No evidence was found that diagnosis or treatment of cutaneous melanoma among long-term survivors has a negative effect on HRQoL.
Although the melanoma patients had, as measured by the overall 15D score as well as several of the dimensions, a slightly lower overall 15D score in comparison to the general population, the difference in the mean scores was not clinically important. Our study found that melanoma patients reported better HRQoL scores at the mental function and discomfort and symptoms dimensions, which is in line with the results of other studies that have found that melanoma survivors may even report better HRQoL scores than the general population (7)

We found that metastatic disease and increasing age contribute to worsening of HRQoL and time elapsed since primary surgery (and thus time since diagnosis) is associated with statistically significant improvement in HRQoL. From these factors, metastatic disease had the largest clinically important effect on 15D scores: the 15D score of patients with metastatic disease was on average 0.049 points lower than that of patients, other things being equal, with no known metastasis at the time of responding to the survey. This result is explained by the variety of symptoms patients with metastatic disease suffer due to treatment side-effects, disease advancement and the psychological stress associated with the awareness of having metastatic disease (13). Our results are consistent with a recently published study, which found that HRQoL in several different types of cancer is lower in a more advanced disease stage than in local disease (14). In previous studies, the results on the association between older age and HRQoL of melanoma patients have been inconsistent, as some studies have found older age to be a contributing factor to lower HRQoL, whereas some studies have found the opposite (7). In our study, increasing age was found to be a contributing factor to lower HRQoL. Improvement in the $15 \mathrm{D}$ score was associated with time elapsed since surgery and thus, since primary diagnosis. 
This is consistent with results from other studies, which have found that impairment in HRQoL is largest during the immediate period after diagnosis (15).

The multivariate analysis showed no statistically significant association of HRQoL with surgical resection margins, which is in agreement with the results of some previous studies (16). Though some studies have found a lower quality of life for patients with larger excision margins, the differences disappear after six months from surgery (12). This may also be the reason why our study did not find a statistically significant decrease in HRQoL for larger excision margins, as the mean time from surgery in our study was markedly longer (6.1 years). Our study also controlled for possible confounders, such as metastatic disease, which may be associated with larger excision margins.

Our study had some limitations that should be noted. Due to the long-time span of our study, a small part of the clinical data was missing from the records and could not be used for analysis. Firstly, out of the 1,052 respondents, 59 patients (5.6\% of the respondents) lacked all clinical data on the melanoma diagnosis from their hospital record and could not be included in the analysis. The remaining 981 patients included in the analysis had at least some clinical data in the hospital records, but for some patients, at least a part of the pathologic report was missing (Table I). For the variables used in the multivariate model, $0.2-2.0 \%$ of the data were missing, depending on the variable. This reflects the changes in melanoma diagnostics and accuracy of the pathologic examination reports throughout the decades. In the multivariate model, the percentage of missing observations was $2.9 \%$. It has been suggested, that less than $5 \%$ of missing data does not bias statistical analysis (17). Consequently, the number of missing observations for the independent variables in the multivariate model in this study can be considered small.

Moreover, data on some important confounding sociodemographic factors such as marital status, employment status, and other comorbidities were not collected. The effect of oncological therapies on HRQoL could have been of interest, but data on this were not collected either. Moreover, due to the retrospective design of our study, no information on HRQoL before diagnosis was available for comparison of HRQoL before and after diagnosis. Our study is also subject to the possibility of selection bias. Out of the 2,075 patients identified from the hospital records, $26.0 \%$ died before the survey was carried out. A further $23.8 \%$ of the 1,381 melanoma patients alive did not answer the questionnaire. As the median 5-year survival rate of metastatic melanoma is only $5 \%$ (18) and our study was conducted up to 24 years after diagnosis, it is likely that patients with the most severe symptoms, and thus the worst HRQoL, had either died before the study was conducted, or if alive, were unable to answer the questionnaire when the questionnaires were sent.
Table II. Multivariate Tobit model results.

\begin{tabular}{lcr}
\hline Variable & $\begin{array}{c}\text { Coefficient } \\
\text { (marginal effect) }\end{array}$ & $p$-Value \\
\hline Constant & 0.857 & $<0.001$ \\
Age & -0.003 & $<0.001$ \\
Gender & 0.007 & 0.264 \\
Complication at any point & -0.020 & 0.070 \\
Metastatic disease at time of response & -0.049 & 0.003 \\
Time from surgery (years) & 0.002 & 0.020 \\
Excision margin <1.00 cm & & \\
$1.00-1.99$ cm & 0.020 & 0.151 \\
$2.00-2.99 \mathrm{~cm}$ & 0.019 & 0.185 \\
$>3.00 \mathrm{~cm}$ & 0.025 & 0.141 \\
Wound closure technique & & \\
Direct closure & 0.009 & 0.342 \\
Skin graft & 0.003 & 0.763 \\
Flap & & \\
Anatomic location: Body & -0.002 & 0.835 \\
Head and neck & 0.005 & 0.558 \\
Upper limb & -0.000 & 0.959 \\
Lower limb & 0.002 & 0.838 \\
Primary SNB & -0.023 & 0.062 \\
\hline
\end{tabular}

*Dependent variable: $15 \mathrm{D}$ score. $\mathrm{n}=953$.

Other studies have found that complete lymph node dissection and sentinel node biopsy may cause significant morbidity due to lymphedema (19). In our multivariate model, a clinically important deteriorating effect of complete lymph node dissection on the 15D score was found but the effect was not statistically significant. Only $23.5 \%$ of our study population had undergone sentinel-node biopsy, most likely due to the timing of our study. The data were collected in 2005 when sentinel node biopsy had only recently been introduced to the national treatment guidelines. The questions associated with sentinel node procedures could be better investigated in a more recently treated study population.

It can be inferred from our results that long-term survival following diagnosis and treatment of non-metastatic melanoma does not have a negative impact on long-term quality of life. For this study, a generic instrument was chosen because it allows for comparison between patients and the general population as well as patients with other diseases. Using a generic instrument has, however, some limitations. It can be anticipated that localized skin cancer and its treatment do not have a significant effect on many of the dimensions of the $15 \mathrm{D}$ instrument. We, however, also collected HRQoL data using the cancer-specific EORTCQLQ-C30 instrument for the same patient cohort and will compare the results obtained with a generic and diseasespecific instrument in a subsequent study.

Until recently, the median survival rate for metastatic melanoma has been only 6-9 months after onset of metastasis, 
and the treatment of metastatic melanoma was in most cases palliative. In addition to surgery and radiation therapy, treatment options for metastatic melanoma have included immunotherapy (interferon alfa-2b, interleukin-2) and cytotoxic chemotherapy. Latest treatment guidelines have adopted new treatment strategies, such as immunotherapy, that utilize antibodies that bind to checkpoint inhibitors of T-cell activation, as well as kinase inhibitors as first-line treatment options for metastatic melanoma (20). Because our study cohort was gathered in 2005, and mainly consisted of patients with localized disease, we were unable to evaluate the effects of these newer systemic therapies. For the same reason, we were also unable to effectively evaluate the effects of complete lymph node dissection on HRQoL.

However, even with the introduction of sentinel-node biopsy and complete lymph node dissection, as well as the new systemic drugs into melanoma treatment guidelines, the primary treatment of melanoma remains, even in the changing landscape of treatments, the excision of the cutaneous tumor. It has also recently been discovered that complete lymph node dissection does not lead to improved survival rates (21), and the attitude towards lymph node procedures is therefore again becoming more conservative. Therefore, even with a relatively old study sample, the results of this study are still relevant. Our study highlights the HRQoL of melanoma patients after long-term survivorship and localized disease. More information on HRQoL of melanoma patients, in particular on those undergoing new therapies for metastatic disease, is needed especially for evaluating health technologies and deciding on health care resource allocation.

\section{Authors' Contributions}

Tiina Jahkola, Pirjo Räsänen, Minna-Liisa Luoma and Risto P. Roine conceived and designed the study. Pia Mylläri, Tiina Jahkola, Pirjo Räsänen and Risto P. Roine collected the data. Pia Heino and Harri Sintonen performed the data analysis. Pia Heino wrote the paper with the assistance of Risto P. Roine and Harri Sintonen.

\section{Acknowledgements}

The Authors would like to thank Marja Nurmi and Anu Roine for their technical assistance in the data collection.

\section{References}

1 Karim-Kos HE, de Vries E, Soerjomataram I, Lemmens V, Siesling S and Coebergh JW: Recent trends of cancer in Europe: a combined approach of incidence, survival and mortality for 17 cancer sites since the 1990s. Eur J Cancer 44(10): 1345-1389, 2008. PMID: 18280139. DOI: 10.1016/j.ejca.2007.12.015

2 de Vries E, Bray FI, Coebergh JW and Parkin DM: Changing epidemiology of malignant cutaneous melanoma in Europe 1953-1997: Rising trends in incidence and mortality but recent stabilizations in western Europe and decreases in Scandinavia. Int J Cancer 107(1): 119-126, 2003. PMID: 12925966. DOI: $10.1002 / \mathrm{ijc} .11360$
3 Cohn-Cedermark G, Rutqvist LE, Andersson R, Breivald M, Ingvar C, Johansson H, Jönsson PE, Krysander L, Lindholm C and Ringborg U: Long term results of a randomized study by the Swedish Melanoma Study Group on 2-cm versus 5-cm resection margins for patients with cutaneous melanoma with a tumor thickness of 0.8-2.0 mm. Cancer 89(7): 1495-1501, 2000. PMID: 11013363.

4 Khayat D, Rixe O, Martin G, Soubrane C, Banzet M, Bazex JA, Lauret P, Vérola O, Auclerc G, Harper P and Banzet P: Surgical margins in cutaneous melanoma $(2 \mathrm{~cm}$ versus $5 \mathrm{~cm}$ for lesions measuring less than 2.1-mm thick). Cancer 97(8): 1941-1946, 2003. PMID: 12673721. DOI: 10.1002/cncr.11272

5 Melanoma. Current Care Guidelines. Working group set up by the Finnish Medical Society Duodecim and the Finnish Cardiac Society. Helsinki: The Finnish Medical Society Duodecim, 2017. Available in Finnish at: http://www.kaypahoito.fi/web/kh/ suositukset/suositus?id=hoi50038 Last accessed April 17, 2019.

6 Miller KD, Siegel RL, Lin CC, Mariotto AB, Kramer JL, Rowland JH, Stein KD, Alteri R and Jemal A: Cancer treatment and survivorship statistics, 2016. CA Cancer J Clin 66(4): 271289, 2016. PMID: 27253694. DOI: 10.3322/caac.21349

7 Hamel JF, Pe M, Coens C, Martinelli F, Eggermont A, Brandberg $\mathrm{Y}$ and Bottomley A: A systematic review examining factors influencing health related quality of life among melanoma cancer survivors. Eur J Cancer 69: 189-198, 2016. PMID: 27838512. DOI: 10.1016/j.ejca.2016.10.008

8 Sintonen H: The 15D instrument of health-related quality of life: properties and applications. Ann Med 33(5): 328-336, 2001. PMID: 11491191.

9 Alanne S, Roine RP, Rasanen P, Vainiola T and Sintonen H: Estimating the minimum important change in the 15D scores. Qual Life Res 24(3): 599-606, 2015. PMID: 25145637. DOI: 10.1007/s11136-014-0787-4

10 Tobin J: Estimation of relationships for limited dependent variables. Econometrica 26(1): 24-36, 1958.

11 Austin PC, Escobar M and Kopec JA: The use of the Tobit model for analyzing measures of health status. Qual Life Res 9(8): 901-910, 2000. PMID: 11284209.

12 Newton-Bishop JA, Nolan C, Turner F, McCabe M, Boxer C, Thomas JM, Coombes G, A'Hern RP nad Barrett JH: A quality-oflife study in high-risk (thickness $>=$ or $2 \mathrm{~mm}$ ) cutaneous melanoma patients in a randomized trial of $1-\mathrm{cm}$ versus $3-\mathrm{cm}$ surgical excision margins. J Investig Dermatol Symp Proc 9(2): 152-159, 2004. PMID: 15083783. DOI: 10.1046/j.1087-0024. 2003.09118.x

13 Kelly B, Raphael B, Smithers M, Swanson C, Reid C, McLeod $\mathrm{R}$, Thomson D and Walpole E: Psychological responses to malignant melanoma. An investigation of traumatic stress reactions to life-threatening illness. Gen Hosp Psychiatry 17(2): 126-134, 1995. PMID: 7789783.

14 Kokkonen K, Tasmuth T, Lehto JT, Kautiainen H, Elme A, Jääskeläinen AS and Saarto T: Cancer patients' symptom burden and health-related quality of life (HRQoL) at Tertiary Cancer Center from 2006 to 2013: A cross-sectional study. Anticancer Res 39(1): 271-277, 2019. PMID: 30591468. DOI: 10.21873/ anticanres. 13107

15 Al-Shakhli H, Harcourt D and Kenealy J: Psychological distress surrounding diagnosis of malignant and nonmalignant skin lesions at a pigmented lesion clinic. J Plast Reconstr Aesthet Surg 59(5): 479-486, 2006. PMID: 16749193.

16 Bergenmar M, Mansson-Brahme E, Hansson J and Brandberg Y: Surgical resection margins do not influence health related quality 
of life or emotional distress in patients with cutaneous melanoma: Results of a prospective randomised trial. Scand J Plast Reconstr Surg Hand Surg 44(3): 146-155, 2010. PMID: 20459368. DOI: $10.3109 / 02844311003791136$

17 Schafer JL: Multiple imputation: a primer. Stat Methods Med Res 8(1): 3-15, 1999. PMID: 10347857. DOI: 10.1177/09622 8029900800102

18 Erdei $\mathrm{E}$ and Torres SM: A new understanding in the epidemiology of melanoma. Expert Rev Anticancer Ther 10(11): 1811-1823, 2010. PMID: 21080806. DOI: 10.1586/era.10.170

19 Wrightson WR, Wong SL, Edwards MJ, Chao C, Reintgen DS, Ross MI, Noyes RD, Viar V, Cerrito PB and McMasters KM: Complications associated with sentinel lymph node biopsy for melanoma. Ann Surg Oncol 10(6): 676-680, 2003. PMID: 12839853.

20 Dummer R, Hauschild A, Lindenblatt N, Pentheroudakis G and Keilholz U: Cutaneous melanoma: ESMO Clinical Practice Guidelines for diagnosis, treatment and follow-up. Ann Oncol 26(5): v126-32, 2015. PMID: 26314774. DOI: 10.1093/ annonc/ mdv297
21 Leiter U, Stadler R, Mauch C, Hohenberger W, Brockmeyer N, Berking C, Sunderkötter C, Kaatz M, Schulte KW, Lehmann P, Vogt T, Ulrich J, Herbst R, Gehring W, Simon JC, Keim U, Martus $\mathrm{P}$ and Garbe C: Complete lymph node dissection versus no dissection in patients with sentinel lymph node biopsy positive melanoma (DeCOG-SLT): a multicentre, randomised, phase 3 trial. Lancet Oncol 17(6): 757-767, 2016. PMID: 27161539. DOI: $10.1016 /$ S1470-2045(16)00141-8
Received February 19, 2019

Revised April 18, 2019

Accepted April 19, 2019 